\title{
ERRATA
}

\section{Fusobacterium simiae, a New Species from Monkey Dental Plaque}

JØRGEN SLOTS AND THOMAS V. POTTS

Department of Oral Biology and Periodontal Disease Clinical Research Center, State University of New York at Buffalo, Buffalo, New York 14226

Volume 32, no. 2, p. 193, Table 3, footnote $a$, line 2: "Lactate was not utilized" should read "Lactate was utilized (propionate produced from lactate)."

\section{Staphylococcus auricularis sp. nov.: an Inhabitant of the Human External Ear

\author{
WESLEY E. KLOOS AND KARL H. SCHLEIFER
}

Department of Genetics, North Carolina State University, Raleigh, North Carolina 27650, and Lehrstuhl für Mikrobiologie, Technische Universität München, D-8000 Munich 2, Federal Republic of Germany

Volume 33, no. 1, p. 13, Table 3, column 4, boxhead: "Aerobic growth" should read "Anaerobic growth." 